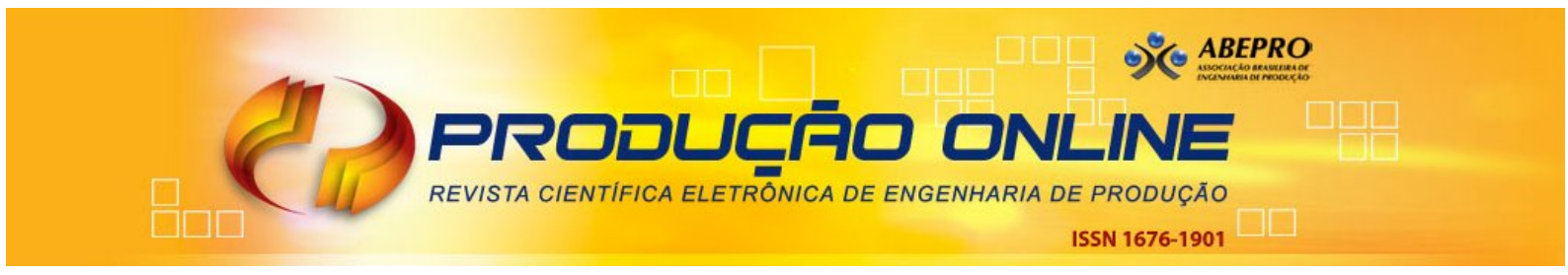

\title{
A PESQUISA EM TRADE-OFFS DE CUSTOS LOGÍSTICOS: ESTUDO BIBLIOMÉTRICO NO PERÍODO DE 2006 A 2016
}

\section{THE RESEARCH IN LOGISTICS COST TRADE-OFFS: BIBLIOMETRIC STUDY IN THE PERIOD FROM 2006 TO 2016}

\author{
Luciano Wallace Gonçalves Barbosa* E-mail: luciano.wallace@hotmail.com \\ Clárison Gonçalves Gamarano*E-mail: clarisongoncalves@gmail.com \\ Ana Luiza Cordeiro Pereira* E-mail: analuiizacordeiro@yahoo.com.br \\ Renata Veloso Santos Policarpo* E-mail: renataveloso@ifmg.edu.br \\ Sílvia Maria Santana Mapa* E-mail: silvia.mapa@ifmg.edu.br \\ * Instituto Federal de Minas Gerais (IFMG), Campus Congonhas, MG
}

\begin{abstract}
Resumo: A otimização dos custos e o acréscimo de benefícios contraídos pelo processo logístico se dão a partir da identificação e da exploração dos trade-offs de custos logísticos. Nesse sentido, o presente trabalho se propõe a apresentar, por meio da metodologia de estudo bibliométrico, a evolução e as principais práticas utilizadas na gestão destes custos nas organizações por meio da avaliação de trade-offs em processos logísticos. Para o estudo, foram levantados sessenta e quatro artigos científicos publicados no período de 2006 a 2016 em periódicos classificados pelo Qualis 2014 e ranqueados entre A1 e B2, disponibilizados na Plataforma Sucupira. Os artigos coletados foram pesquisados nas áreas de Engenharias III, da qual a Engenharia de Produção faz parte, e nas Ciências Sociais Aplicadas, onde o tema custos logísticos também é amplamente abordado. A pesquisa realizou-se em três etapas: coleta de dados, análise e síntese. Os resultados revelaram que a área de Pesquisa Operacional lidera como ciência empregada ao utilizar métodos descritivos e estocásticos. Enquanto aspecto restritivo, ficou evidente a dificuldade em aplicações diretas em tradeoffs de custos logísticos, o que caracteriza uma lacuna de estudos no que diz respeito à integração entre a logística e a contabilidade.
\end{abstract}

Palavras-chave: Logística. Custos Logísticos. Trade-offs. Trade-offs de Custos Logísticos. Bibliometria.

\begin{abstract}
The optimization of costs and the increase of benefits acquired by the logistical process are given from the identification and exploitation of trade-offs of logistics costs. In this sense, this paper aims to present, through the bibliometric study methodology, the evolution and the main practices used in the management of these costs in organizations through the evaluation of trade-offs in logistics processes. For the study, sixty-four scientific articles were collected, including articles published in the period from 2006 to 2016 in periodicals classified by the Qualis 2014 and ranked between A1 and B2, available on the Sucupira Platform. The collected articles were searched in the areas of Engineering III, of which the Production Engineering is part, and in the Applied Social Sciences area, in which the logistical costs theme is also widely approached. The research was carried out in three stages: data collection, analysis and synthesis. The results revealed that the area of Operational Research leads as the employed science by using descriptive and stochastic methods. As a restrictive aspect, it was evident the difficulty in direct applications to logistics costs in trade-offs, which characterizes a study gap with regard to the integration between logistics and accounting.
\end{abstract}

Keywords: Logistics. Logistics Costs. Trade-offs. Logistics Cost Trade-offs. Bibliometrics.

Revista Produção Online. Florianópolis, SC, v. 18, n. 2, p. 641-664, 2018. 


\section{INTRODUÇÃO}

Cada vez mais as empresas têm buscado vantagens competitivas a fim de aumentarem sua participação no mercado e os retornos acima da média. Os gestores , por sua vez, ficam incumbidos por oportunidades de redução de custos no contexto das áreas administrativas sob sua responsabilidade. Nessa perspectiva, o mapeamento e análise dessas áreas tornam-se funções importantes dentro das organizações. De forma geral, quando se mapeia essas áreas administrativas, encontram-se oportunidades algumas vezes subjugadas ao segundo plano. Uma dessas áreas é a logística. Algumas vezes despercebida pelos gestores, os quais a conduz de forma intrínseca, a logística detém o poder de otimizar os portfólios empresariais, e consequentemente contribuir para a redução do custo total empresarial.

Diante da necessidade dos gestores buscarem meios para atuar na redução de custos sem afetar a eficiência produtiva, os gestores logísticos têm dado maior relevância para a necessidade de determinação e avaliação dos custos logísticos empresariais, que são detentores de parcelas significativas do custo total de produção. Algumas técnicas, como a análise de trade-offs ou até mesmo a seleção correta de método de custeio, ainda que não muito aderidas pelas empresas, podem oferecer vantagens expressivas na busca pela contração de despesas e melhoria do desempenho econômico-financeiro das empresas.

A análise de trade-offs apresenta uma oportunidade de desmistificar concepções genéricas, como as que apontam quais cortes de custos implicam em redução de qualidade, ou que a redução do nível de inventários acarreta em aumento no custo de transportes. Assim, a apreciação dos trade-offs se faz a partir da mensuração de trocas compensatórias entre os custos logísticos e a entrega ao cliente, de modo a promover uma relação de ganha-ganha em que os custos são reduzidos ao passo que o nível de serviço é mantido ou até mesmo melhorado em consequência de ações coletivas.

Nesse sentido, o presente trabalho se propõe a apresentar, por meio da metodologia de estudo bibliométrico, a evolução e as principais práticas utilizadas na gestão de custos logísticos nas organizações a partir da aplicação da avaliação de 
trade-offs em processos logísticos. Para esse estudo, foram levantados sessenta e quatro artigos científicos, publicados no período de 2006 a 2016 em periódicos classificados pelo Qualis 2014 e ranqueados entre A1 e B2, disponibilizados na Plataforma Sucupira. Os artigos coletados foram pesquisados nas áreas de Engenharias III, da qual a Engenharia de Produção faz parte e, nas Ciências Sociais Aplicadas, em que o tema custos logísticos também é amplamente abordado.

\section{FUNDAMENTAÇÃO TEÓRICA}

\subsection{Logística}

Por ser uma atividade que está presente em quase todas as empresas, seja em grande ou pequena proporção, a logística tem se destacado como uma das principais atividades administrativas, isso porque os custos incorporados são relevantes e ocorre a aplicação de técnicas que melhoram o seu desempenho, que por sua vez, acarretam em resultados notavelmente positivos. Em um mundo majoritariamente urbano, a movimentação de produtos e serviços coloca-se como elemento de essencial importância para a sociedade (SILVA, 2013), elemento esse que, segundo Faria e Costa (2011), ficou muito tempo como atividade relegada a um segundo plano pelas empresas, considerada apenas como uma função de apoio.

Christopher (2007) define logística como sendo o gerenciamento estratégico da compra, transporte e armazenagem de produtos, sendo matéria-prima, acabados e semiacabados, e suas informações, ao mesmo passo que Bowersox et al. (2006), afirmam que a logística se refere ao planejamento e à gestão de sistemas orientados ao controle do transporte e da localização geográfica dos estoques de materiais, visando à minimização dos custos. Ballou (2012) destaca em sua obra, que grande parte das empresas de serviços, agências e instituições, tanto governamentais quanto privadas, necessitam do auxílio de um especialista em logística. Ao afirmar a presença massiva dessa técnica nas empresas, destaca-se que, ao contrário do que muitos pensam, a logística não é somente o transporte dos suprimentos da cadeia produtiva, mas envolve todo um conjunto de atividades e processos, os quais se somam em busca de uma rede integrada de abastecimento e movimentação, com 
práticas que aperfeiçoam toda a cadeia logística, acrescentando aos produtos valores de lugar, tempo, qualidade e informação (NOVAES, 2007).

Como objetivos principais da logística, Faria e Costa (2011) afirmam ser o provimento ao cliente de níveis de serviços por eles requeridos, com a entrega do produto certo, no lugar, momento, condições e custos certos. Alguns clientes podem custar mais que outros, então a logística pode utilizar o princípio da exclusividade para identificar os custos desses clientes e, então, perceber se os negócios com esses clientes são rentáveis, em vista dos custos gerados com os mesmos (GOMES; RIBEIRO, 2011).

Novaes (2007) apresenta a evolução da Logística a partir de quatro fases, onde é detalhado o encadeamento ocorrido até a conceituação moderna do gerenciamento da cadeia de suprimentos. Essas fases são resumidas a seguir:

(i) primeira fase: corresponde ao período pós Segunda Guerra Mundial, marcado pela intensificação de processos de produção em série, sendo o estoque o elemento chave no balanceamento da cadeia produtiva. Nessa fase, observa-se baixo índice de integração, e uma visão técnica e operacional.

(ii) segunda fase: marcada pela introdução da customização, aumento da oferta de produtos, flexibilização de processos produtivos e utilização da multimodalidade no transporte de mercadorias. Inicia-se a utilização de sistemas de informações, porém a integração é rígida e ainda há limitada visão estratégica por parte dos gestores.

(iii) terceira fase: reconhece-se uma integração flexível e dinâmica entre os agentes da cadeia de suprimentos onde as informações são monitoradas em tempo real por via eletrônica. Acrescenta-se conteúdo estratégico e as empresas e a preocupação com a satisfação do cliente.

(iv) quarta fase: ocorre um salto qualitativo onde a questão logística é tratada de forma estratégica e se busca soluções que promovam competitividade, troca de informações e consolidação de alianças estratégicas. A procura por excelência torna-se parâmetro essencial para crescimento do negócio.

A logística não trata somente de uma atividade, mas sim, de uma sequência de atividades, denominadas atividades logísticas e que, segundo Ballou (2012), estão separadas em dois grandes grupos: as atividades primárias (principais) e as atividades secundárias (de apoio). Nas atividades principais, encontram-se aquelas 
de maior importância nas empresas e com custos mais expressivos no orçamento final, sendo essas relacionadas o transporte, estoque e processamento de pedidos. Já as atividades de apoio vêm somar aos principais valores imprescindíveis, mas seus custos são menos relevantes que as demais, são elas: manutenção da informação, programação do produto, obtenção, manuseio, embalagem e armazenagem.

Consoante Amaral e Guerreiro (2014), a variação de custos em uma atividade causa alteração em uma ou mais atividades logísticas, fato que se destaca a importância da análise conjunta de todos os fatores, assim como confirmado pelo estudo de caso feito por Silva et al. (2013), que concluem que a caracterização das atividades logísticas do setor estudado mostrou que mais de sessenta por cento das variáveis analisadas são de suma importância na qualidade logística das indústrias.

\subsection{Custos logísticos}

Partindo do pressuposto de que a logística está inserida em todas as atividades de uma organização, os gerentes têm observado a gestão de custos logísticos como uma ferramenta estratégica para obter e sustentar as vantagens competitivas da organização (GONÇALVES, 2015). Dessa maneira, é necessário que se trabalhe continuamente na busca de recursos que promovam a redução dos custos e garantam a excelência no âmbito da prestação de serviços aos clientes.

Valendo-se disso, Castiglioni e Nascimento (2014) defendem que um dos principais trade-offs da logística consiste em proporcionar maior nível de serviço sem que isso acarrete em acréscimo substancial aos custos da empresa. Ou seja, a gestão de custos logísticos consiste em uma ferramenta de ganho de mercado por meio do mapeamento dos custos, ao passo que se otimiza o controle das operações e viabiliza a tomada de decisões na organização de forma mais consistente.

Para acolher a esse propósito, Castiglioni (2013) alerta que considerar apenas o custo de transporte como essencial é um erro fatal, uma vez que esse representa somente um dos elementos que compõem os custos logísticos. Dessa forma, o autor apresenta quatro fatores prioritários que devem ser considerados para análise, sendo eles: (i) custos com armazenagem, (ii) custos com processamento de pedidos, (iii) custos com estoque e (iv) custos com transporte. 
Em conformidade, Campos e Brasil (2013) advogam que a otimização dos custos logísticos deve, inclusive, atentar para as exigências do mercado, cada vez mais volátil. Nesse sentido, a gestão de custos deve buscar princípios como: maior disponibilidade de produtos e serviços, redução do prazo de entrega e maior facilidade na colocação de pedidos. Ainda segundo os mesmos autores, deve-se avaliar a participação de mercado e considerar o respectivo volume de investimentos com perspectivas e cenários de longo prazo.

Finalmente Pozo (2004) defende a utilização do conceito de Valor Agregado, na avaliação dos custos logísticos, conceito este relacionado à percepção que um stakeholder tem sobre um nível de serviço logístico apresentado. Consoante, o autor sugere que o método ABC (Activity-Based Costing) corresponde à ferramenta de gerenciamento mais eficaz na gestão dos custos logísticos, uma vez que este se propõe a eliminar distorções causadas na apuração dos custos dos produtos e serviços, objetivando melhor precisão do custeio de produtos e serviços. Ou seja, o caráter quantitativo do método $A B C$ torna-se, dentro deste contexto, um componente-chave para a análise e avaliação de processos logísticos, além de melhorar a qualidade das decisões.

\subsection{Trade-offs de custos logísticos}

De acordo com Amaral e Guerreiro (2014), trade-off refere-se à troca compensatória entre a perda em algum aspecto e o ganho em outro. Com relação aos trade-offs de custos logísticos, os autores afirmam que compreendem as trocas compensatórias em que o acréscimo em um custo logístico pode ser compensado pela redução em outro custo logístico e/ou pelo aumento do nível de serviço oferecido ao cliente. Lambert e Armitage (1979) ressaltam que a existência dos trade-offs faz com que cortes isolados de custos sejam responsáveis pelo aumento do custo total e piora do nível de serviço ao cliente, e que a avaliação dos trade-offs tem por objetivo identificar as reações de cada componente logístico às ações propostas, a fim de mensurar as compensações e escolher a alternativa que otimiza o custo total e o desempenho econômico-financeiro.

Castiglioni e Nascimento (2014) afirmam que um dos principais trade-offs de um sistema logístico consiste em proporcionar maior nível de serviço sem resultar 
em aumento substancial dos custos da organização. Blecic et al. (2007) ressaltam em seus estudos a importância dos trade-offs entre o nível de serviço e os custos de manutenção de estoques, uma das principais atividades da logística, a qual segundo Ballou (2012) agrega valor de tempo. Amaral e Guerreiro (2014) enfatizam por sua vez, os trade-offs presentes entre as atividades de manutenção de inventários e transportes, sendo que os custos relacionados às duas atividades são os mais relevantes, proporcionalmente ao total de custos logísticos. Kator (2007), Chow (2008), Murphy e Poist (2007) afirmam que os trade-offs entre as atividades supracitadas são relevantes, diante da análise do desenho das redes logísticas, e tais áreas são importantes quanto às habilidades logísticas requeridas pelos gestores.

Janssen (2006) e Torabi et al. (2015) por meio de suas pesquisas chamam a atenção para a importância da análise dos trade-offs de custos logísticos e afirmam que para que as empresas obtenham sucesso é preciso que todas as organizações envolvidas na cadeia de suprimentos tenham um conhecimento preciso e analisem os trade-offs, a fim de melhor controlarem as atividades logísticas e consequentemente reduzirem seus custos, os quais, como já exposto por Bowersox e Closs (2014), mostram-se de forma relevante diante dos custos totais de uma instituição. Cheong e Song (2013), por meio de seus estudos, enfatizam que uma logística precisa, mesmo que impacte nos custos, garante confiabilidade ao sistema, agrega valor e aumenta os lucros potencialmente, o que caracteriza assim importante trade-off a ser analisado.

\section{METODOLOGIA}

Esse estudo consiste em uma pesquisa quantitativa e qualitativa que, segundo Fonseca (2002), se centra na objetividade e recorre à linguagem matemática para descrever as causas de um fenômeno, as relações entre variáveis, etc. O método utilizado foi a revisão bibliométrica, que permite encontrar uma quantidade de periódicos essenciais, que se supõe possuir os artigos mais relevantes publicados sobre determinado assunto, e se dá através do estudo dos aspectos quantitativos da produção, disseminação e uso da informação publicada (CUNHA, 1985; CASTRO, 2001; ARAÚJO e ARENCIBIA; VANTI, 2002). 
A pesquisa realizou-se em três etapas: coleta de dados, análise e síntese (CROSSAN e APAYDIN, 2010; KEUPP, PALMIE e GASSMANN, 2012). A coleta de dados se deu pela pesquisa de artigos publicados entre os anos 2006 e 2016 em periódicos do Qualis 2014, classificados entre A1 e B2, na Plataforma Sucupira. Foram identificados sessenta e quatro artigos das áreas de Engenharias III, da qual a Engenharia de Produção faz parte e, das Ciências Sociais Aplicadas, em que o tema custos logísticos também é abordado.

A fase de análise iniciou-se com a seleção dos estudos que tratavam especificamente sobre trade-offs de custos logísticos, sendo identificados sessenta e quatro artigos. A partir de tais estudos, foram produzidos fichamentos em que se abordaram os objetivos, principais tópicos da literatura, principais autores, metodologias utilizadas e resultados obtidos. Por meio dos fichamentos, foi construída uma tabela com todos os dados obtidos, em que se adicionou o país e universidade dos autores dos artigos.

Os dados identificados e agrupados na tabela passaram então por uma análise de conteúdo que compreende técnicas de pesquisa que permitem, de forma sistemática, a descrição das mensagens relacionadas ao contexto da enunciação, bem como as inferências sobre os dados coletados; portanto pode ser utilizada para o aprofundamento de estudos quantitativos e qualitativos (CAVALCANTE et. al, 2014; BARDIN, 1977). Assim, os dados foram classificados e sintetizados de acordo com suas linhas de pesquisas, metodologias, locais de estudo, objetivos dos estudos, casos estudados, resultados, ano de publicação e classificação Qualis. Tais análises foram então evidenciadas como resultados da análise bibliométrica realizada.

\section{RESULTADOS}

Na sequência são apresentados os resultados da pesquisa, embasados nos dados quantitativos que geraram a análise qualitativa. As estratificações são feitas em subtópicos, conforme segue. 


\subsection{Análise das Metodologias}

As metodologias utilizadas nos artigos analisados foram classificadas em conformidade com a taxonomia proposta por Fillipini (1997), que segundo Saraiva Júnior (2010) é indicada para estudos realizados no campo acadêmico do periódico "Gestão de Produção e Operações". De acordo com a taxonomia de Fillipini (1997), bem como com as descrições de Berto e Nakano (1998), pode-se classificar as metodologias em: modelagem matemática/quantitativa, que diz respeito ao uso de equações matemáticas para descrever o funcionamento de um sistema produtivo; simulação, que se refere à utilização de técnicas computacionais para simular o funcionamento de um sistema por meio de modelos matemáticos; survey, com o uso de um instrumento de coleta de dados, através de técnicas de amostragem e análise estatística aplicados a amostras extensas; estudo de caso, análise aprofundada de um ou mais casos caracterizada pela interação entre o pesquisador e a pesquisa; teórico/conceitual, que possui como enfoque discussões conceituais a partir da literatura; e experimentação, que aborda a relação causal entre duas variáveis do sistema sob condições controladas pelos pesquisadores.

Ao fazer-se uso das classificações citadas acima, percebe-se pela Figura 1 as metodologias encontradas e a representatividade de artigos em que as mesmas são utilizadas.

Figura 1 - Representatividade das metodologias identificadas

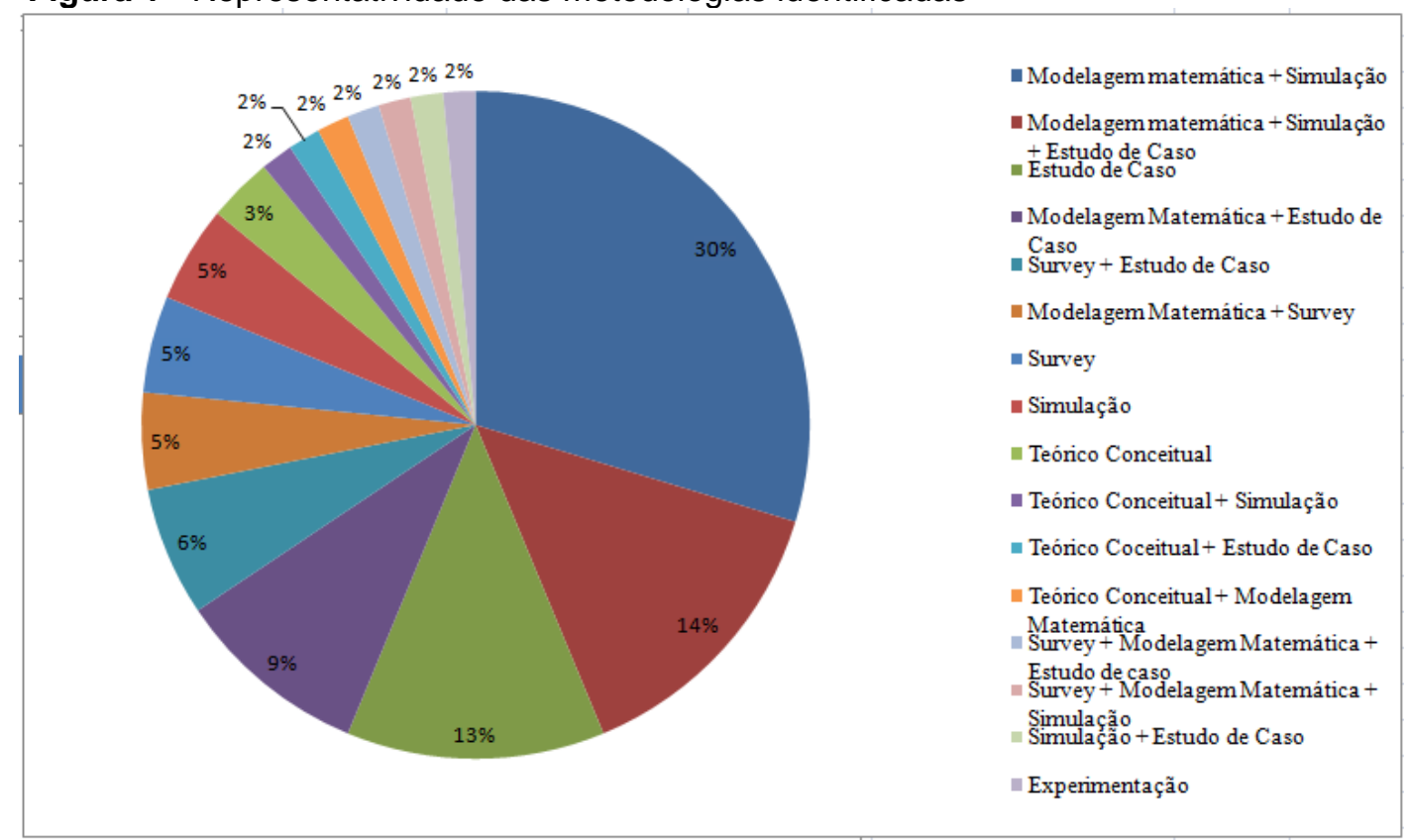

Fonte: Dados da pesquisa 
Por meio da Figura 1 infere-se a predominância dos artigos que utilizaram a Modelagem Matemática juntamente com a Simulação, os quais caracterizam cerca de $30 \%$ dos estudos. Ao estratificar as metodologias abordadas, pode-se afirmar que o método mais utilizado é a Modelagem Matemática, que aparece em 62,5\% dos artigos; seguido de: Simulação, encontrada em 53,1\% dos estudos; Estudo de Caso, utilizado em 46,9\% das pesquisas; Survey, aplicado a 18,8\% dos estudos; Teórico Conceitual, que aparece em 6,3\% dos artigos e Experimentação, observado em 1,56\% dos estudos analisados; como exposto no Tabela 1. É importante ressaltar que alguns artigos utilizaram metodologias combinadas, ou seja, mais de um método na pesquisa, motivo pelo qual a soma das metodologias ultrapassa o total de artigos analisados.

Tabela 1 - Estratificação das metodologias identificadas

\begin{tabular}{ccc} 
Metodologia & Número de artigos & Frequência \\
\hline Modelagem Matemática & 40 & $62,5 \%$ \\
Simulação & 34 & $53,1 \%$ \\
Estudo de Caso & 30 & $46,9 \%$ \\
Survey & 12 & $18,8 \%$ \\
Teórico Conceitual & 4 & $6,3 \%$ \\
Experimentação & 1 & $1,56 \%$ \\
\hline
\end{tabular}

Fonte: Dados da pesquisa

Por fim, infere-se a predominância de pesquisas de caráter quantitativo. Pode-se perceber também a complexidade dos estudos abordados, principalmente os que apresentam como metodologia a modelagem matemática e simulação, os quais segundo Gavira (2003) necessitam de conhecimento técnico prévio e conciso acerca do tema e variáveis tratadas para serem desenvolvidos. Ademais, a complexidade dos estudos é coerente com o nível de artigos abordados na bibliometria, os quais foram publicados em periódicos conceituados pelo Qualis 2014.

\subsection{Principais locais de estudo}

Por meio da Tabela 2, percebe-se a predominância de publicações nos Estados Unidos. Tal fato pode associar-se aos Estados Unidos serem uma das maiores economias do mundo, investindo assim em técnicas de otimização de processos e estudos de trade-offs e logística. 
Tabela 2 - Estratificação dos países de publicação dos artigos

\begin{tabular}{ccc}
\hline Países & Quantidade & Artigos \\
\hline Estados Unidos & 8 & $12.5 \%$ \\
Irã & 6 & $9.4 \%$ \\
India & 5 & $7.8 \%$ \\
Canadá & 4 & $6.3 \%$ \\
China & 4 & $6.3 \%$ \\
Taiwan & 4 & $6.3 \%$ \\
Brasil & 3 & $4.7 \%$ \\
Holanda & 3 & $4.7 \%$ \\
Itália & 3 & $4.7 \%$ \\
Singapura & 3 & $4.7 \%$ \\
Tailândia & 3 & $4.7 \%$ \\
Alemanha & 2 & $3.1 \%$ \\
Austria & 2 & $3.1 \%$ \\
ReinoUnido & 2 & $3.1 \%$ \\
Suécia & 2 & $3.1 \%$ \\
Belgica & 1 & $1.6 \%$ \\
Coréia do Sul & 1 & $1.6 \%$ \\
Dinamarca & 1 & $1.6 \%$ \\
Filândia & 1 & $1.6 \%$ \\
Hong Kong & 1 & $1.6 \%$ \\
Japão & 1 & $1.6 \%$ \\
Macau & 1 & $1.6 \%$ \\
México & 1 & $1.6 \%$ \\
Portugal & 1 & $1.6 \%$ \\
Turquia & 1 & $1.6 \%$ \\
Total & $\mathbf{6 4}$ & $\mathbf{1 0 0 . 0 \%}$ \\
\hline
\end{tabular}

Fonte: Dados da pesquisa

Observa-se também maior número de publicações em outros países desenvolvidos ou emergentes, com grande volume de produção de bens ou muito populosos. O Irã, por exemplo, tem uma economia mista de planejamento centralizado, propriedade estatal do petróleo e de outras empresas grandes, comércio e agricultura tradicional e serviços privados de pequeno porte. Já o Canadá concentra uma associação de desenvolvimento econômico e um alto índice de contratempos causados por adversidades climáticas, fator que acrescenta uma variável extra no que diz respeito ao planejamento logístico. Além disso, corresponde a um país com grande extensão territorial.

A presença dos países populosos, como Estados Unidos, Irã, Índia, China e Brasil, remetem à necessidade destes em atender às necessidades logísticas próprias, comuns às regiões com grande aglomeração de atividades e pessoas. A partir de tal análise, pode-se encontrar similaridades nas características de tais países onde os artigos foram publicados com o conteúdo das publicações. Isso 
significa que independente da extensão territorial, a alta taxa de ocupação por indústrias influencia diretamente no processo de gestão dos trade-offs.

\subsection{Principais objetivos dos estudos}

Fazendo-se a leitura dos trabalhos citados na metodologia, percebe-se certo direcionamento nos objetivos de tais obras. Como principal ponto, destaca-se a importância de analisar a relação entre trade-off e a conveniência do cliente. Outro objetivo foco é minimizar os custos logísticos analisando a relação entre oferta e demanda. Muitas vezes, o aumento de custos em algum aspecto diminui custos em outros. Por isso, necessita-se fazer uma análise criteriosa e cautelosa da situação investigada.

Todos os outros pontos têm uma relação direta com o objetivo citado anteriormente. Para tal análise, devem-se avaliar os atuais desafios nos sistemas fabris e de logística, enfrentando assim os desafios na elaboração de políticas logísticas. Para que tais desafios sejam enfrentados, torna-se importante capacitar os profissionais em relação aos trade-offs de custos, para que assim tomem decisões corretas no que se refere à seleção de parceiros, fornecedores, exclusividade na rede de negócios, melhores rotas, entre outros.

No que se diz respeito às melhores rotas, deve-se considerar também o horário ideal para transporte e as questões ambientais, como menor consumo de combustível, menor emissão de $\mathrm{CO}_{2}$ e, consequentemente, menor tempo de operação.

Em alguns casos, faz-se o estudo do controle logístico específico para determinada atividade, considerando também aspectos como a flexibilidade logística, a segmentação ou adaptação do produto ou atividade ao mercado. Em outros casos, há decisões que consideram critérios múltiplos, multiprodutos com diferentes tipos de instalações, multiperíodo ou multimodalidade. Por isso, torna-se importante a utilização de modelos de tomada de decisão, como Ótimos de Pareto, Teoria dos Jogos, modelos estocásticos, entre outros (CAMBERO e SOWLATI, 2016; HUANG et al., 2016; CHEONG e SONG, 2013). Desse modo, tais ferramentas podem ser aplicadas em inúmeras situações como seleção de fornecedores, seleção 
de modo de transporte, estratégias de preços sobre lucros, informações sobre incertezas e emissões de poluentes.

Ademais, não se podem esquecer outros fatores importantes que devem ser apontados, como qualidade, quantidade, custo de inventário a partir de estoques, o desempenho do ERP (Enterprise Resourcing Planning) utilizado, a logística reversa, a importância dos trade-offs logísticos no comércio internacional etc. Por fim, vê-se a importância dos trade-offs no custo logístico total, por isso faz-se indispensável o uso de estratégias de controle, para que assim não haja prejuízos no processo e esse possa cada vez mais ser otimizado.

\subsection{Principais casos estudados}

Dentre os trabalhos estudados, muitos apresentaram-se como estudos de modelos de otimização e acompanhamento de suas evoluções entre outras estratégias e softwares. Atentou-se também para métodos de configuração de frotas no que se diz respeito ao tamanho, custos (como custos com estoques e inventários, por exemplo), maximização de lucros e segurança. Alguns estudos de casos usaram como exemplo algumas empresas logísticas que estão em ampla expansão em alguns países, como a United Parcel Service - UPS.

Entretanto, vários outros artigos trouxeram problemas diferentes que aumentam a visão das pessoas acerca da magnitude da logística e do trade-off em diferentes setores. Tal prática se faz muito importante no setor financeiro de empresas, pois a logística leva em conta o fluxo de produtos, serviços e informação, os quais influenciam diretamente nas finanças destas. Como exemplos de ramos empresarias que aplicam tais conhecimentos em diversas de suas áreas, tem-se as companhias aéreas e automáticas, o setor aeroespacial, centros de serviços eletrônicos, fábricas de produtos plásticos, embalagens, roupas, alimentos etc. A ATeT, uma grande companhia americana de telecomunicações, por exemplo, utiliza de ERP em seu sistema logístico, otimizando seus processos e serviços (CHAN et al., 2012).

Tais estudos de casos fizeram menções importantes no que se diz respeito ao desempenho social dos trabalhadores nas empresas e na importância de trade-offs logísticos na avaliação de profissionais que entram no mercado, pois estes querem 
crescer cada vez mais e obter reconhecimento pelo trabalho prestado. Atentou-se também às redes de negócios inteligentes e à gestão da cadeia de suprimentos como uma forma de concorrência e crescimento no mercado, pois as empresas que não se voltam para tais práticas permanecem estagnadas e, consequentemente, perdem competitividade.

Atualmente, empresas que não dão créditos às questões ambientais do mesmo modo, acabam perdendo oportunidades nos negócios (NOVAES, 2007). É fundamental tratar de um melhor uso dos recursos e avaliar os impactos destes e dos seus processos no meio ambiente, fazendo assim uma análise dos seus ciclos de vida, ou seja, considerando todos os níveis de emissões de poluentes e impactos ambientais associados. Aqueles que se preocupam com os assuntos sustentáveis fazem valer o sentido do selo verde, já que a expansão da consciência coletiva com relação ao meio ambiente tem induzido um novo posicionamento por parte dos empresários e executivos em face dessas questões, inclusive com relação à logística (TACHIZAWA, 2015).

Há também algumas ocorrências de suma relevância, como o fornecimento de emergência de sangue durante catástrofes potenciais. Caso tal fornecimento não fosse estudado em tempo hábil e de maneira otimizada, muitas pessoas morreriam por causa de uma falha logística. Observa-se, portanto, que não são apenas fatos industriais, mas também, fatos relacionados à sobrevivência e a saúde das pessoas que cerceiam a logística. Por isso é importante também um gerenciamento de risco, onde são avaliadas as causas e consequências de um sistema mal programado, contemplando particularidades, como a produção em escala, canais de distribuição, análise de trade-offs, sensibilidade da oferta de preços para demandas e ofertas dinâmicas e problemas de localização.

Os sistemas logísticos também carecem de ser usados na eliminação de fraudes que aumentam a concorrência desleal e diminuição da qualidade, como na pirataria marítima, que causa problemas de abastecimento (SPERANZA FILHO, 2014). Nesta e em outras situações o governo e o setor privado devem utilizar técnicas relativas ao tema em estudo para suprimir tais entraves e cada vez mais criar cenários nos quais haja harmonia no mercado interno e também externo. Trade-offs são importantes no âmbito nacional e internacional, atendendo às 
tendências de um mercado globalizado, elemento imprescindível na procura de soluções logísticas (AMARAL; GUERREIRO, 2014).

\subsection{Principais resultados}

De acordo com os resultados encontrados na pesquisa, conclui-se que a área da Pesquisa Operacional lidera como ciência empregada na análise de trade-offs de custos logísticos, até mesmo em questões de desempenho geral e diminuição de erros (VAHDANI et al., 2012). Métodos como programação matemática e simulação auxiliam na tomada de decisão pela facilidade de compreender melhor as variáveis e questões importantes no serviço logístico, permitindo trade-offs que diminuam os custos e aumentem o nível de serviço (PAIVA et al., 2009; BRAEKERS et al., 2016). $\mathrm{Na}$ pesquisa, foram vistas aplicações de modelos matemáticos e simulação na eficácia de recuperação integrada de voos, deslocamento de transporte de materiais recicláveis, problemas de transporte, a cadeia de suprimentos da indústria do vestuário, entre outros (JAKHAR, 2015; XIAOYUN BING et al., 2015; YUZHEN HU et al., 2016).

Algumas obras analisadas mostraram que a utilização de outros tipos de programação, como algoritmos heurísticos e programação por metas, podem ser integradas em pesquisas com maior diversidade de variáveis, aumentando a qualidade do resultado devido a flexibilidade de programação. Softwares de otimização como o CPLEX resultaram algumas soluções matematicamente ótimas com programações exatas. Mas, em todas as situações analisadas, deve-se levar em conta que os modelos propostos devem ser adaptados à realidade de cada empresa, onde também devem ser deixadas de lado influências emocionais e culturais de modo que essas variáveis não interfiram na solução gerada pelos modelos computacionais.

Análises de sensibilidade geradas em modelos matemáticos demonstram que a redução de custos nem sempre condiz com melhor taxa de utilização dos recursos, cabendo uma análise qualitativa para possíveis trade-offs nos modelos de decisão (MEHRDAD et al., 2012). Quanto aos riscos, segundo Mohammad et al. (2015), modelos matemáticos comprovaram que, no caso de fornecimentos, é 
menos arriscado quando se utiliza mais de um fornecedor, evitando um possível monopólio de abastecimento.

Dentro dos modelos propostos foram feitas adaptações, as quais permitiram aos pesquisadores analisarem a eficiência de trade-offs no âmbito de custos operacionais, principalmente no quesito de investimento em tecnologia da informação para otimizar processos. Um exemplo clássico citado por Castillo-Villar et al. (2012) é quando se aumentam custos com softwares informáticos para geração de rotas logísticas que mantenham nível de serviço exigido pelos clientes. Esses mesmos softwares integram processos decisórios e permitem explorar inter-relações entre entidades empresariais, dentro da cadeia de suprimentos. O ERP (Enterprise Resourcing Planning) é outro exemplo de investimento resultante em otimização de processos e redução de custos.

A respeito da atividade transportes, estudos compararam variáveis significativas na atividade logística, como clientes, custos e tempo médio de entrega, onde se concluiu que o modelo de produção-entrega associado com maiores quantidades de remessa e menores números de viagem reduzem em cerca de seis a nove por cento do custo total de transportes. Nesse caso, a variação da redução do custo está relacionada pela necessidade do cliente, de modo a atender o nível de serviço logístico esperado. Composições de modelos de custos logísticos não são fixas, ou seja, uma implementação bem-sucedida requer análise de uma série de trade-offs para alcançar um controle da logística em todos os níveis e atividades da cadeia de suprimentos.

Redes de negócio inteligentes na logística também foram citadas por alguns autores (PAU, 2012; MOHAMMAD et al., 2015). Elas permitem configuração de processos e stakeholders sobre o serviço, implicando em mudanças tanto técnicas quanto da natureza do negócio, oferecidas através de um conjunto de ideias e proposições. Custos de manutenção também foram incorporados, balanceando as compras e encomendas de suas empresas de acordo com a taxa de defeitos dos produtos e os custos de pós-venda gerados pelas garantias.

Outro importante ponto abordado pelos autores (TZENG et al., 2012; BING et al., 2015) diz respeito a trocas entre os custos logísticos nos quesitos de nível de serviço prestado e impactos ambientais, tendo sido desenvolvida uma metodologia com eficiência comprovada por uma empresa chinesa, onde se conseguiu balancear 
a redução de custos logísticos juntamente com os danos causados ao meio ambiente.

Quanto aos profissionais logísticos, a pesquisa concluiu que estes sabem da existência de trade-offs de custos logísticos, mas não possuem um entendimento amplo sobre esse conceito, em especial da participação destes no custo total de produção (AMARAL et al., 2014; SINHA et al., 2016). Interessante ponto levantado na pesquisa trata de trade-offs da empresa com seus stakeholders e até mesmo entre outras empresas do mesmo ramo, como um benchmarking, principalmente quando se trata de seleção de colaboradores, uma vez que estes são importantes ativos nas organizações e precisam de atenções específicas para melhor desenvolver seus papéis e aumentar a capacidade de desempenho das empresas (SOHN et al., 2015).

\subsection{Relação publicações $X$ ano}

A figura 2 apresenta a relação da quantidade de artigos analisados, estratificados pelos anos de publicação. Pelo gráfico, observa-se que as publicações sobre o assunto tiveram aumento a partir do ano de 2011, tendo 2015 com maior número de publicações, e os demais anos com aproximadamente sete trabalhos, em média. A partir disso, conclui-se que o tema tem tido sua importância acentuada, mas ainda necessita de mais pesquisas publicadas com a finalidade de troca de conhecimentos entre as organizações acerca de suas aplicações de trade-offs na composição dos custos logísticos. 
Figura 2 - Estratificação das publicações por ano

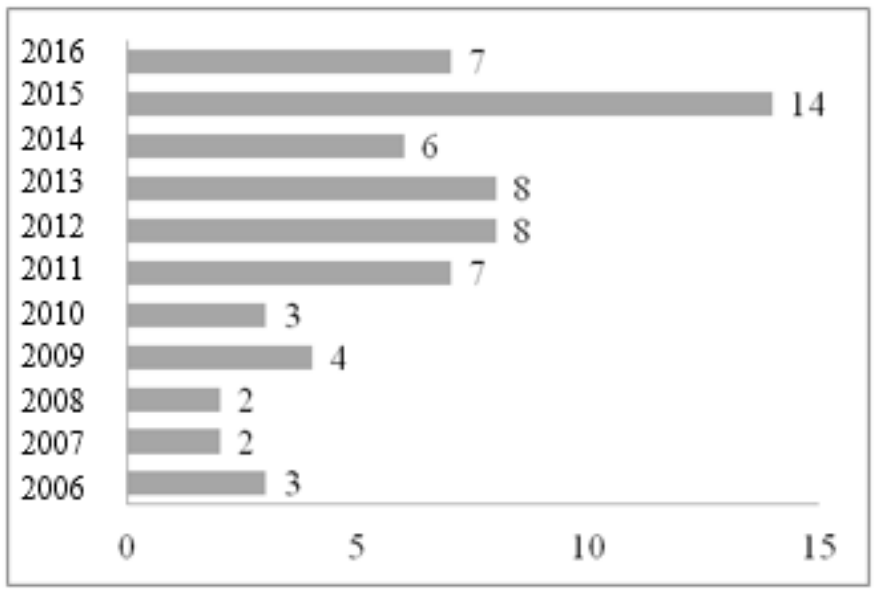

Fonte: Dados da pesquisa

\subsection{Relação Qualis dos artigos}

Na Figura 3, observam-se os Qualis CAPES dos artigos pesquisados na bibliometria, para as áreas: Engenharias III e Ciências Sociais Aplicadas. A maior parte dos artigos está indexada em periódicos com conceito A, tendo quase $70 \%$ dos trabalhos publicados em revistas A1 e A2, sendo esta com maior representatividade quantitativa dos artigos. Já os periódicos com qualis B têm representatividade menor, com 20 artigos publicados em revistas com conceito B1 e B2. A partir dessas informações, infere-se a importância do tema, uma vez que os trabalhos são publicados em importantes periódicos, principalmente em periódicos internacionais.

Figura 3 - Estratificação dos qualis das obras analisadas

$$
\text { - } 1 \text { 1 }=\mathrm{A} 2 \square \mathrm{B} 1 \square \mathrm{B} 2
$$

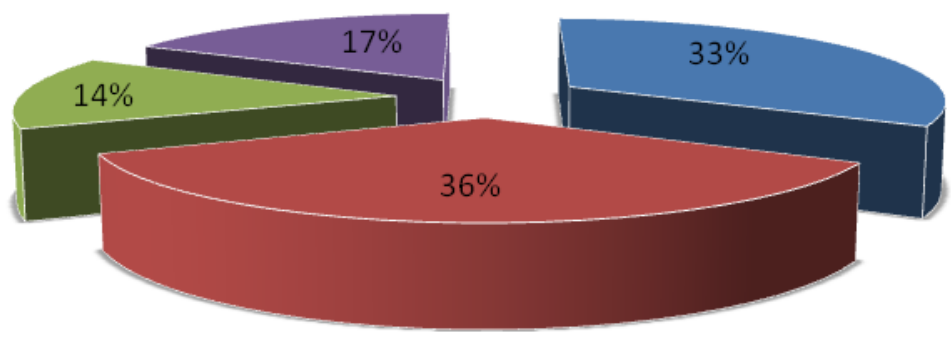

Fonte: Dados da pesquisa 
Os periódicos com maior número de trabalhos selecionados para a presente pesquisa foram: Journal of Logistics Systems and Management; European Journal of Operational Research; Transportation Research Part E: Logistics and Transportation Review; Journal of computational and applied mathematics; Business Process Management Journal; Applied Mathematical Modelling.

\section{CONCLUSÕES}

O trabalho atingiu os objetivos propostos de realizar uma bibliometria com a finalidade de compreender o estado da literatura na temática de trade-offs de custos logísticos através de pesquisa e análise, tanto qualitativa quanto quantitativa, de artigos publicados em congressos nacionais e internacionais.

Os resultados mostraram a predominância de ciências, como a Pesquisa Operacional, na resolução de problemas que envolvem a troca compensatória entre atividades dentro das empresas, utilizando-se de métodos descritivos e estocásticos. Ainda foram citadas outras metodologias no campo da ciência da administração, como o benchmarking, análise de stakeholders, processo de agregação de valor, entre outros. Todas as técnicas apresentaram bons resultados, principalmente quando combinadas a fim de alcançar diferentes objetivos, convergindo a um ideal principal.

Com a pesquisa, conseguiu-se concluir que a maior parte das pesquisas é aplicada em empresas de vários lugares do mundo, com resultados positivos quanto às metodologias aplicadas nos estudos de caso. A principal lacuna existente, percebida na realização da bibliometria, é a aplicação específica de trade-offs em custos logísticos, uma vez que estes são aplicados de forma implícita dentro de atividades que compõem as operações das empresas, ou seja, os trabalhos não tratam especificamente de trade-offs de custos logísticos, mas sim de trocas compensatórias em atividades que são alocadas na logística, como transporte, embalagem, aquisições, entre outras.

Além disso, observou-se que apesar do avanço no que concerne à aplicação de modelos que promovam os trade-offs de custos logísticos, em muitas situações as empresas ainda se encaixam na terceira fase da logística devido à dificuldade de 
compreender a magnitude de tal aplicação, cujo ganho se dá em uma perspectiva geral e não por cortes individuais. Outros elementos que dificultam a análise dos trade-offs dizem respeito ao adequado aproveitamento de softwares de gestão de custos e o ajustamento de informações contábeis.

\section{REFERÊNCIAS}

AMARAL, J. V. Trade-offs de custos logísticos. 2012. Dissertação (Mestrado em Controladoria e Contabilidade: Contabilidade) - Faculdade de Economia, Administração e Contabilidade, Universidade de São Paulo, São Paulo, 2012. DOI: http://dx.doi.org/10.11606/D.12.2012.tde-12112012-142501

AMARAL, J. V.; GUERREIRO, R. Conhecimento e Avaliação dos Trade-offs de Custos Logísticos: um Estudo com Profissionais Brasileiros. Rev. contab. finanç., São Paulo, v. 25, n. 65, p. 111-123, agosto 2014.

DOI: $\underline{\text { http://dx.doi.org/10.1590/S1519-70772014000200003 }}$

AMARAL, J. V.; GUERREIRO, R. O custo total e as soluções logísticas. Revista Ambiente Contábil - UFRN. Natal-RN, v. 6, n. 1, p. 64-82, jan./jun 2014.

AMIT SINHA, W. P.; MILLHISER, Y. H. Matching supply with demand in supply chain management education. The International Journal of Logistics Management, vol. 27, $\mathrm{n}$. 3, p.837-861, 2016. DOI: https://doi.org/10.1108/IJLM-03-2015-0058

ARAUJO RUIZ, J. A.; ARENCIBIA JORGE, R. Informetría, bibliometría y cienciometría: aspectos teórico-prácticos. ACIMED, Ciudad de La Habana, v. 10, n. 4, p. 5-6, agosto 2002.

BALLOU, R. H. Logística empresarial: transportes, administração de materiais e distribuição física. 1. ed. São Paulo: Atlas, 2012.

BARDIN, L. Análise de conteúdo. Lisboa: Edições 70, 1977.

BERTO, R. M. V. B.; NAKANO, D. N. Métodos de pesquisa na Engenharia de Produção. In: ENCONTRO NACIONAL DE ENGENHARIA DE PRODUÇÃO, 18. Niterói, 1998. Anais. Niterói: UFF/ABEPRO, 1998. (CDROM)

BING, B.; BLOEMHOF-RUWAARD, J.; CHAABANE, A.; VORST, J. Global reverse supply chain redesign for household plastic waste under the emission trading scheme. Journal of Cleaner Production, vol. 30, p.1-12, 2015.

DOI: https://doi.org/10.1016/j.jclepro.2015.02.019

BLECIC, I.; CECCHINI, A.; TRUNFIO, G. A. A decision support tool coupling a causal model and a multi-objective genetic algorithm. Appllntell, vol. 26, p. 125-137, 2007.

DOI: https://doi.org/10.1007/11504894 88

BOWERSOX, D. J.; CLOSS, D. J.; COOPER, M. B. Gestão logística de cadeia de suprimentos. Porto Alegre: Bookman, 2006. 529 p. 
BOWERSOX, D. J.; CLOSS, D. J.; COOPER, M. B.; BOWERSOX, J. C. Gestão e Logística da Cadeia de Suprimentos. 4. ed. São Paulo: Bookman, 2014.

BRAEKERS, K.; HARTL, R. F.; PARRAGH, S. N.; TRICOIRE, F. A bi-objective home care scheduling problem: Analyzing the trade-off between costs and client inconvenience.

European Journal of Operational Research, vol. 248, n. 2, p. 428-444, jan. 2016.

DOI: https://doi.org/10.1016/j.ejor.2015.07.028

CAMBERO, C., e SOWLATI, T. Incorporating social benefits in multi-objective optimization of forest-based bioenergy and biofuel supply chains. Applied Energy, v. 178, p. 721-735, 2016. DOI: https://doi.org/10.1016/j.apenergy.2016.06.079

CAMPOS, F. R.; BRASIL, V. M. Logística: teia de relações. 1. ed. São Paulo: Intersaberes, 2013.

CASTIGLIONI, J. A. Logística operacional: Guia Prático. 3. ed. São Paulo: Érica, 2013.

CASTIGLIONI, J. A.; NASCIMENTO F. C. Custos de processos logísticos. 1. ed. São Paulo: Érica, 2014.

CASTILLO-VILLAR, K. K.; SMITH, N. R.; SIMONTON, J. L. A model for supply chain design considering the cost of quality. Applied Mathematical Modelling, v. 36, p. 5920-5935, dez. 2012. DOI: https://doi.org/10.1016/i.apm.2012.01.046

CAVALCANTE, R. B.; CALIXTO, P.; PINHEIRO, M. M. Análise de Conteúdo: considerações gerais, relações com a pergunta de pesquisa, possibilidades e limitações do método. Inf. eSoc.:Est., João Pessoa, v. 24, n.1, p. 13-18, jan./abr. 2014.

CHAN, X.; LAU, Y.; NG, J. M. J. Critical evaluation of ERP implementation on firm performance: a case study of ATeT. International Journal of Logistics Systems and Management, vol. 12, n. 1, p. 52-69, 2012. DOI: https://doi.org/10.1504/IJLSM.2012.047058

CHEONG, T.; SONG, S. H. The value of information on supply risk under random yields. Transportation Research Part E, vol. 60, p. 27-38, 2013.

DOI: https://doi.org/10.1016/j.tre.2013.09.006

CHOW, G. Getting back to basics. Canadian Transportation Logistics. Don Mills, v. 111, n. 10, p. 40, out. 2008.

CHRISTOPHER, M. Logística e gerenciamento da cadeia de suprimentos: criando redes que agregam valor. São Paulo: Thomson Learning, 2007.

CROSSAN, M. M.; APAYDIN, M. A Multi-Dimensional Framework of Organizational Innovation: A Systematic Review of the Literature. Journal of Management Studies, vol. 47, n. 6, p. 1154-1191, 2010. DOI: https://doi.org/10.1111/j.1467-6486.2009.00880.x

CUNHA, Miriam Vieira da. Os periódicos em ciência da informação: uma análise bibliométrica. Ciência da Informação, v. 14, n. 1, jun. 1985.

DOI: https://doi.org/10.18225/ci.inf..v14i1.225

FARIA, A. C.; COSTA, M. F. G. Gestão de custos logísticos. São Paulo: Atlas, 2011. 
FILIPPINI, R. Operations management research: some reflections on evolution, models and empirical studies in OM. International Journal of Operations and Production

Management, v.17, n.7, p. 655-70, 1997.

DOI: https://doi.org/10.1108/01443579710175583

FONSECA, J. J. S. Metodologia da pesquisa científica. Fortaleza: UEC, 2002.

GAVIRA, M. O. Simulação computacional como uma ferramenta de aquisição de conhecimento. 2003. Dissertação (Mestrado em Engenharia de Produção) Escola de Engenharia de São Carlos, Universidade de São Paulo, São Carlos, 2003.

DOI: https://doi.org/10.11606/D.18.2003.tde-20052003-004345

GOMES, C. F. S.; RIBEIRO, P. C. C. Gestão da cadeia de suprimentos: integrada à tecnologia da informação. Cengage Learning, 2011. 360 p.

DOI: https://doi.org/10.1007/s10489-006-0009-z

GONÇALVES, A. T. P. Estudo sobre custos logísticos: conceitos, classificação e operacionalização. In: ENCONTRO NACIONAL DE PESQUISA EM ADMINISTRAÇÃO, 34, 2015, Belo Horizonte. Anais. Belo Horizonte: EnANPAD, 2015.

HU, Y.; SONG, Y.; ZHAO, K.; XU, B. Integrated recovery of aircraft and passengers after airline operation disruption based on a GRASP algorithm. Transportation Research Part E, vol. 87, p. 97-112, 2016. DOI: https://doi.org/10.1016/j.tre.2016.01.002

HUANG, Y., WANG, K., ZHANG, T., e PANG, C. Green supply chain coordination with greenhouse gases emissions management: a game-theoretic approach. Journal of Cleaner Production, vol. 112, p. 2004-2014, 2016. DOI: https://doi.org/10.1016/j.jclepro.2015.05.137

JAKHAR, S. K. Performance evaluation and a flow allocation decision model for a sustainable supply chain of an apparel industry. Journal of Cleaner Production, vol. 87, p. 391-413, 2015. DOI: https://doi.org/10.1016/j.jclepro.2014.09.089

JANSSEN, M. Insights from the introduction of a supply chain co-ordinator. Business Process Management Journal, vol. 10, n. 3, p. 300 - 310, 2006.

DOI: https://doi.org/10.1108/14637150410539704

KATOR, C. Inventory costs rise dramatically. Modern Materials Handling: Warehousing Management Edition. Framingham, v. 62, n. 07, p. 09, jul. 2007.

KEUPP, M.; PALMIÉ, M.; GASSMANN, O. The strategic management of innovation: A systematic review and paths for future research. International Journal of Management Reviews, vol. 14, n. 4, p. 367-390, 2012.

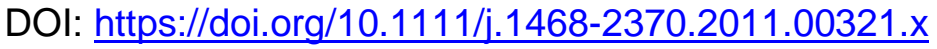

LAMBERT, D. M.; ARMITAGE, H. M. Distribution costs: the challenge: The key to managing the physical distribution function is total cost analysis, rather than haphazard stabs at cutting specific costs. Management Accounting (pre-1986). Montvale, v. 60, n. 11, p. 33- 37, 45, jun. 1979.

MEHRBOD, M.; Tu, N.; MIAO, L.; WENJING, D. Interactive fuzzy goal programming for a multi-objective closed-loop logistics network. Ann Oper Res, vol. 201, p. 367-381, 2012. DOI: https://doi.org/10.1007/s10479-012-1192-4 
MOHAMMAD, A. N.; MOHAMMAD, S.; REZA, H. Supplier selection considering strategic and operational risks: a combined qualitative and quantitative approach. Prod. Eng. Res. Devel, v. 9, p. 665-673, 2015. DOI: https://doi.org/10.1007/s11740-015-0643-6

MURPHY, P. R.; POIST, R. F. Skill requirements of senior-level logistics executives: a mlongitudinal assessment. Supply Chain Management. Bradford, v. 12, n. 6, p. 423-31, 2007. DOI: $\underline{\text { https://doi.org/10.1108/13598540710826353 }}$

NOVAES, A. G. Logística e gerenciamento da cadeia de distribuição: estratégia, operação e avaliação. 3. ed. Rio de Janeiro: Campus, 2007. 400 p.

PAIVA, R. P. O.; MORABITO, R. An optimization model for the aggregate production planning of a Brazilian sugar and ethanol milling company. Ann Oper Res, vol. 169, p. 117130, 2009. DOI: https://doi.org/10.1007/s10479-008-0428-9

PAU, L-F. Smart business networks: interaction-coordination aspects and risks. Business Process Management Journal, v. 18, n. 5, p. 829 -843, 2012.

DOI: https://doi.org/10.1108/14637151211270180

POZO, H. Administração de recursos materiais e patrimoniais: uma abordagem logística. 3. ed. São Paulo: Atlas, 2004.

SILVA, A. N.; LIMA, J. E.; PEREZ, R. Caracterização e desempenho logístico das indústrias laticinistas da Zona da Mata e Campo das Vertentes em Minas Gerais, Brasil. Ciência Rural, v.43, n.7, p.1337-1343, 2013. DOI: http://dx.doi.org/10.1590/S010384782013005000078

SILVA, J. V. R. Mobilidade urbana e entregas ao varejo: proposta de modelo de desempenho da logística de distribuição física. Dissertação (Mestrado em Administração) - Universidade Federal de Minas Gerais, Belo Horizonte, MG, 2013.

SOHN, M.; SOHN, W.; KLAAS-WISSING, T.; HIRSCH, B. The influence of corporate social performance on employer attractiveness in the transport and logistics industry: Insights from German junior talent. International Journal of Physical Distribution e Logistics Management, v. 45, n. 5, p.486 -505, 2015. DOI: https://doi.org/10.1108/IJPDLM-07-2014-0150

SPERANZA FILHO, N. Os Custos da Pirataria Marítima à Economia Global. In: Profa. Dra. Eliane M. Octaviano Martins. (Org.). Direito Marítimo e Portuário: questões fundamentais. 1ed. Santos: Leopoldianum, v. 1, p. 4-4, 2014.

TACHIZAWA, T. Gestão ambiental e responsabilidade social corporativa: estratégias de negócios focadas na realidade brasileira. 8. ed. São Paulo: Atlas, 2015.

TORABI, S. A.; BAGHERSAD, M.; MANSOURI, S. A. Resilient supplier selection and order allocation under operational and disruption risks. Transportation Research Part E, vol. 79, p. 22-48, 2015. DOI: https://doi.org/10.1016/j.tre.2015.03.005

TZENG, G.; HUANG, C. Combined DEMATEL technique with hybrid MCDM methods for creating the aspired intelligent global manufacturing e logistics systems. Ann Oper Res, vol. 197, p.159-190, 2012. DOI: https://doi.org/10.1007/s10479-010-0829-4 
VAHDANI, B.; IRANMANESH, S. H.; MOUSAVI, S. M.; ABDOLLAHZADE, M. A locally linear neuro-fuzzy model for supplier selection in cosmetics industry. Applied Mathematical Modelling, vol. 36, p. 4714-4727, 2012. DOI: https://doi.org/10.1016/j.apm.2011.12.006

VANTI, N. A. P. Da bibliometria à webometria: uma exploração conceitual dos mecanismos utilizados para medir o registro da informação e a difusão do conhecimento. $\mathbf{C i}$.

Inf., Brasília, v. 31, n. 2, p. 369-379, agosto 2002. DOI:

http://dx.doi.org/10.1590/S0100-19652002000200016

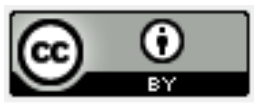

Artigo recebido em 23/05/2017 e aceito para publicação em 11/08/2017

DOI: http://dx.doi.org/10.14488/1676-1901.v18i2.2882 